\title{
Mosaic trisomy 9
}

INSERM

\section{Source}

INSERM. (1999). Orphanet: an online rare disease and orphan drug data base. Mosaic trisomy 9. ORPHA:99776

Mosaic trisomy 9 is a rare chromosomal anomaly syndrome, with a highly variable phenotype, principally characterized by intellectual disability, growth and developmental delay, facial dysmorphism (incl. microphthalmia, deep-set eyes, low-set, malformed ears, bulbous nose, high-arched palate, micrognathia) and congenital heart defects (e.g. ventricular septal defect), as well as urogenital (e.g. hypoplastic genitalia, cryptorchidism), skeletal (congenital joint dislocations or hyperflexion, scoliosis/kyphosis) and central nervous system anomalies (hydrocephalus, Dandy-Walker malformation). Pigmentary mosaic skin lesions along the lines of Blaschko are also frequently observed. 